\title{
Effect of Packing and Storage Behaviour on Shelf Stability of Functionally Enriched Fruit Rolls
}

\author{
K. D. Sharma, Vinay Chandel, Anil Gupta* and Anil K. Verma
}

Dept. of Food Science and Technology, Dr. Y.S. Parmar University of Horticulture and Forestry, Nauni, Solan, H.P. (173 230), India

\section{Corresponding Author}

Anil Gupta

e-mail: anilguptafst@gmail.com

\author{
Article History \\ Article ID: IJEP0226 \\ Received in $27^{\text {th }}$ October, 2017 \\ Received in revised form $1^{\text {st }}$ February, 2018 \\ Accepted in final form $10^{\text {th }}$ February, 2018
}

\begin{abstract}
Storage stability of apple, persimmon, peach and pear fruit rolls prepared by incorporating outer peel of fruits after packing in laminated pouches(150 gauge) and polythene pouches (200 gauge) and storage under refrigerated and ambient temperature was evaluated. The packing of fruit rolls in laminated pouches followed by storage under refrigerated conditions $\left(4-5^{\circ} \mathrm{C}\right)$ upto six months resulting in minimum changes in quality as compared to ambient temperature. During 6 months of storage, an increase in mean moisture content from 13.43 to $15.43 \%$ was noticed in polythene pouches whereas, decrease in moisture content from 13.43 to $13.35 \%$ was noticed in laminated pouches. After 6 months of storage, ascorbic acid decreased from 14.27 to 13.83 , total phenols from 810.12 to $755.24 \mathrm{mg} 100 \mathrm{~g}^{-1}$, free

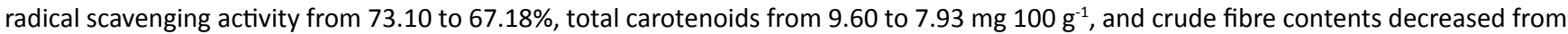
1.75 to $1.38 \%$ irrespective of fruit roll type and storage temperature. The packing of fruits rolls in laminated pouches followed by storage at refrigerated conditions is optimized for storage of fruit rolls.
\end{abstract}

Keywords: Fruit rolls, laminated pouches, storage, scavenging activity, phenols, crude fibre

\section{Introduction}

Apple, persimmon, peach and pear are the most important fruits grown in the temperate regions of the world. Interestingly, it has been observed that outer peel of fruits is rich in poly phenolic compounds as compared to pulp. These fruits contain $80-92 \%$ pulp with peel and $74-87 \%$ pulp without peel, with highest pulp percentage with peel and without peel of $92 \%$ and $87 \%$ in persimmon, followed by $90 \%$ and $85 \%$ in apple, $84 \%$ and $75 \%$ in pear respectively. According to Chinnici et al. (2004), the peel of apple is rich in phenolics and the integrated peel has the highest total antioxidant capacity (18.56 $\mu \mathrm{M} \mathrm{kg}^{-1}$ fresh weight). The persimmon fruits are found to be rich source of ascorbic acid (7.11 $\mathrm{mg} \%)$, total phenols (693 mg \%) beside having good free radical scavenging activity (84.53\%), followed by apple (total phenol $364 \mathrm{mg} \%$, free radical scavenging activity $75.30 \%$ ) and peach (total phenol $309.46 \mathrm{mg} \%$, free radical scavenging activity $68.86 \%$ ) (Sharma et al., 2014). Further, preparation of fruit bar and leather from different fruits has been reported as papaya leather (Chan and Caveletto, 1987), sapota and jackfruit leather (Che and Taufik, 1995), durian leather (Irwandi et al., 1998), guava leather (Vijayanand et al., 2000), and fruit bars from plum pulp (Thakur, 1997). However, the systematic information on preparation and storage behaviour of functionally enriched fruit rolls is scanty. Therefore, the present investigation was planned to study the storage behaviour of functionally enriched fruit rolls.

\section{Materials and Methods}

\subsection{Preparation of single fruit rolls}

Fruit rolls were prepared using apple, persimmon, peach and pear fruits by incorporating outer peel of fruits followed by drying in cabinet drier $\left(60 \pm 2{ }^{\circ} \mathrm{C}\right)$. Among different pretreatments, treatment $\mathrm{T}_{3}\left(25^{\circ} \mathrm{B}\right.$ TSS) in apple, pear and peach and $\mathrm{T}_{4}\left(30^{\circ} \mathrm{B}\right.$ TSS $)$ in case of persimmon was recorded best and thus optimized for development of single fruit rolls on basis of sensory evaluation (Sharma et al., 2014). The samples ranked best on basis of sensory evaluation and quality were selected for storage under refrigerated temperature $\left(4-5{ }^{\circ} \mathrm{C}\right)$ and ambient temperature $\left(20-24{ }^{\circ} \mathrm{C}\right)$ after packing in laminated (150 gauge) and polythene pouches (200 gauge).

\subsection{Analysis}

Different chemical characteristics were determined by following standard methods at 0, 3 and 6 months storage intervals. Total sugars in percent were estimated by following method detailed by Lane and Eynon, (1923). Total phenols content was extracted in $80 \%$ ethanol and was estimated on the basis of their reaction with an oxidizing agent in FolinCiocalteau reagent under alkaline conditions (Bray and Thorpe, 1954). Total carotenoids were estimated and expressed 
as $\beta$-carotene $\mathrm{mg} 100 \mathrm{~g}^{-1}$ sample (Ranganna 1997). Crude fibre in percent $(w / w)$, ascorbic acid, crude protein and the moisture content (\%) in single fruit rolls were determined by using standard analytical methods (AOAC, 1995) while free radical scavenging activity was measured by method detailed by Brand et al. (1995). Sensory evaluation of samples was done by a semi-trained panel of experts, on basis of nine points (9) hedonic scale method (Amerine et al., 1965). The overall acceptability of developed products was measured by evaluating products on colour, texture and taste basis during 6 months of storage.

\subsection{Equilibrium relative humidity (ERH)}

For determining the equilibrium relative humidity of fruit rolls the samples were spread evenly on Petri dishes, which were then placed inside the desiccators containing $\mathrm{H}_{2} \mathrm{SO}_{4}$ solutions of varying concentrations representing relative humidity levels ranging between $0-100 \%$. The loss or gain in weight of each sample was recorded at 24 hours intervals till the samples attained the equilibrium. After equilibrium, data was plotted against the respective relative humidity to determine ERH of given sample (Ranganna, 1997). Critical and danger points of rolls were calculated according to the weight equilibrium method (Wink, 1946).

\subsection{Statistical analysis}

All the data were analyzed in 3 replications and average value was taken for further representation. Data were analyzed by using completely randomized design (CRD) (Panse and Sukhatme, 1967).

\section{Results and Discussion}

\subsection{Chemical and nutritional quality characteristics}

The mean moisture content of singe rolls increased to $14.39 \%$ after six months of storage from the initial $13.43 \%$ moisture irrespective of fruit roll type and packing material (Table 1). Maximum moisture content was recorded in persimmon (15.16\%) with minimum in pear $(11.31 \%)$ fruit rolls and after the six months of storage increased to 15.75 and 11.82 percent, respectively. The packaging material also shows significant effect on moisture contents of stored rolls as the fruit rolls packed in polyethylene pouches absorbed maximum moisture during storage (13.43 to $15.43 \%$ ) whereas rolls in laminated pouches shows a slight decrease in moisture content from 13.43 to $13.35 \%$. Therefore, the laminated pouches are better packaging material due to their barrier properties as reported by Ambrose and Sreenarayana (1998). Further, the fruit rolls stored at low temperature shows a marginal change in moisture content (13.43 to $13.63 \%)$ as compared to ambient temperature (13.43 to $14.35 \%)$. The results follows the same trends as reported by Mir (1990) in mango bars, Bhardwaj and Lal (1990); Kumar (1999); Mishra et al. (2002) in osmotically dehydrated apricots, dried apples

Table 1: Changes in moisture content (\%) of single fruit rolls during storage

\begin{tabular}{|c|c|c|c|c|c|c|c|c|c|c|c|c|c|c|c|}
\hline \multirow{3}{*}{$\begin{array}{l}\text { Packing } \\
\text { Material } \\
\text { (P) }\end{array}$} & \multirow[t]{3}{*}{$\mathrm{SP}$} & \multicolumn{12}{|c|}{ Fruit (F) } & \multirow{3}{*}{$\begin{array}{l}\text { Grand } \\
\text { mean }\end{array}$} & \multirow{3}{*}{$\begin{array}{c}\text { Mean } \\
\text { (S) }\end{array}$} \\
\hline & & \multicolumn{3}{|c|}{ Apple } & \multicolumn{3}{|c|}{ Peach } & \multicolumn{3}{|c|}{ Pear } & \multicolumn{3}{|c|}{ Persimmon } & & \\
\hline & & AT & RT & Mean & AT & RT & Mean & AT & RT & Mean & AT & RT & Mean & & \\
\hline \multicolumn{16}{|c|}{ Polyethylene } \\
\hline \multirow[t]{3}{*}{ Pouches } & 0 & 14.18 & 14.18 & 14.18 & 13.06 & 13.06 & 13.06 & 11.31 & 11.31 & 11.31 & 15.16 & 15.16 & 15.16 & 13.43 & 13.43 \\
\hline & 3 & 16.85 & 14.55 & 15.70 & 15.52 & 13.54 & 14.53 & 13.52 & 11.73 & 12.63 & 17.78 & 15.76 & 16.77 & 14.91 & 14.14 \\
\hline & 6 & 17.59 & 14.88 & 16.24 & 16.35 & 13.93 & 15.14 & 14.26 & 12.06 & 13.16 & 18.23 & 16.13 & 17.18 & 15.43 & 14.39 \\
\hline Mean & & 16.21 & 14.54 & 15.37 & 14.98 & 13.51 & 14.24 & 13.03 & 11.70 & 12.37 & 17.06 & 15.68 & 16.37 & 14.59 & \\
\hline \multirow[t]{3}{*}{ LP } & 0 & 14.18 & 14.18 & 14.18 & 13.06 & 13.06 & 13.06 & 11.31 & 11.31 & 11.31 & 15.16 & 15.16 & 15.16 & 13.43 & \\
\hline & 3 & 14.12 & 14.15 & 14.14 & 12.99 & 13.02 & 13.01 & 11.25 & 11.28 & 11.27 & 15.11 & 15.13 & 15.12 & 13.38 & \\
\hline & 6 & 14.09 & 14.13 & 14.11 & 12.97 & 13.00 & 12.99 & 11.20 & 11.25 & 11.23 & 15.07 & 15.12 & 15.10 & 13.35 & \\
\hline \multicolumn{2}{|l|}{ Mean } & 14.13 & 14.15 & 14.14 & 13.01 & 13.03 & 13.02 & 11.25 & 11.28 & 11.27 & 15.11 & 15.14 & 15.13 & 13.39 & \\
\hline \multicolumn{2}{|c|}{ Grand mean } & 15.17 & 14.35 & 14.76 & 13.99 & 13.27 & 13.63 & 12.14 & 11.49 & 11.82 & 16.09 & 15.41 & 15.75 & 13.99 & \\
\hline \multicolumn{2}{|l|}{ Mean $(\mathrm{T})$} & 14.35 & 13.63 & & & & & & & & & & & & \\
\hline \multicolumn{16}{|c|}{$\mathrm{CD}(p=0.05)$} \\
\hline \multicolumn{3}{|l|}{ Fruit (F) } & 0.063 & FxP & 0.094 & \multicolumn{2}{|c|}{ PxS } & 0.085 & \multicolumn{2}{|c|}{ FxTxS } & \multicolumn{3}{|l|}{0.150} & & \\
\hline \multicolumn{3}{|c|}{ Packing $(\mathrm{P})$} & 0.052 & FxT & 0.079 & Tx & kS & 0.071 & \multicolumn{2}{|c|}{ PxTxS } & 0.116 & & & & \\
\hline \multicolumn{3}{|c|}{ Temperature $(T)$} & 0.038 & FxS & 0.112 & FxP & PXT & 0.133 & \multicolumn{2}{|c|}{ FxPxTxS } & 0.260 & & & & \\
\hline \multicolumn{3}{|c|}{ Storage (S) } & 0.058 & PxT & 0.063 & FXP & PxS & 0.189 & & & & & & & \\
\hline
\end{tabular}

SP: Storage Period (S) (Months); LP: Laminated Pouches; AT: Ambient temperature; RT: Refrigerated temperature 
and dehydrated apple rings respectively. However, a decrease in moisture content during storage of jackfruit bars has been reported by Krishnaveni et al., 1999).

Data presented in Table 2 shows that the total sugars in single fruit rolls were in the range of 58.36 to $64.30 \%$ with maximum total sugars in persimmon (64.30\%) and minimum in peach (58.36\%). After six months storage, total sugar contents decreased to $59.32 \%$ from initial $61.12 \%$ irrespective of fruit rolls and storage temperature. Among packaging materials, fruit rolls packed in polyethylene pouches observed maximum decrease in total sugars during storage (61.12 to $59.18 \%$ ) as compared to a slight decrease from an initial value of 61.12 to $59.46 \%$ observed in laminated pouch. Low temperature also shows significant effect on total sugars. Slight increase in reducing sugars of products during storage might be attributed to the inversion of non-reducing sugars and other polysaccharides into reducing sugars. The results are in accordance with the results discussed in dried carrot and apple rings (Sharma et al., 2002; Ambrose and Sreenarayana, 1998) whereas; increase in sugars in dehydrated mango slices and

Table 2: Changes in total sugars (\%) in single fruit rolls during storage

\begin{tabular}{|c|c|c|c|c|c|c|c|c|c|c|c|c|c|c|c|}
\hline \multirow{3}{*}{$\begin{array}{l}\text { Packing } \\
\text { Materia } \\
\text { (P) }\end{array}$} & \multirow[t]{3}{*}{$\mathrm{SP}$} & \multicolumn{12}{|c|}{ Fruit (F) } & \multirow{3}{*}{$\begin{array}{l}\text { Grand } \\
\text { mean }\end{array}$} & \multirow{3}{*}{$\begin{array}{c}\text { Mean } \\
(\mathrm{S})\end{array}$} \\
\hline & & \multicolumn{3}{|c|}{ Apple } & \multicolumn{3}{|c|}{ Peach } & \multicolumn{3}{|c|}{ Pear } & \multicolumn{3}{|c|}{ Persimmon } & & \\
\hline & & AT & RT & Mean & AT & RT & Mean & AT & RT & Mean & AT & RT & Mean & & \\
\hline \multicolumn{16}{|c|}{ Polyethylene } \\
\hline \multirow[t]{3}{*}{ Pouches } & 0 & 60.86 & 60.86 & 60.86 & 58.36 & 58.36 & 58.36 & 60.96 & 60.96 & 60.96 & 64.30 & 64.30 & 64.30 & 61.12 & 61.12 \\
\hline & 3 & 59.76 & 60.30 & 60.03 & 57.31 & 57.83 & 57.57 & 59.86 & 60.40 & 60.13 & 63.13 & 63.71 & 63.42 & 60.29 & 60.43 \\
\hline & 6 & 58.67 & 59.19 & 58.93 & 56.27 & 56.77 & 56.52 & 58.76 & 59.29 & 59.03 & 61.97 & 62.53 & 62.25 & 59.18 & 59.32 \\
\hline Mean & & 59.76 & 60.12 & 59.94 & 57.31 & 57.65 & 57.48 & 59.86 & 60.22 & 60.04 & 63.13 & 63.51 & 63.32 & 60.20 & \\
\hline \multirow[t]{3}{*}{ LP } & 0 & 60.86 & 60.86 & 60.86 & 58.36 & 58.36 & 58.36 & 60.96 & 60.96 & 60.96 & 64.30 & 64.30 & 64.30 & 61.12 & \\
\hline & 3 & 60.04 & 60.58 & 60.31 & 57.57 & 58.09 & 57.83 & 60.14 & 60.68 & 60.41 & 63.43 & 64.00 & 63.72 & 60.57 & \\
\hline & 6 & 58.94 & 59.47 & 59.21 & 56.53 & 57.03 & 56.78 & 59.04 & 59.57 & 59.31 & 62.26 & 62.82 & 62.54 & 59.46 & \\
\hline \multicolumn{2}{|l|}{ Mean } & 59.95 & 60.30 & 60.13 & 57.49 & 57.83 & 57.66 & 60.05 & 60.40 & 60.23 & 63.33 & 63.71 & 63.52 & 60.38 & \\
\hline \multicolumn{2}{|c|}{ Grand mean } & 59.86 & 60.21 & 60.03 & 57.40 & 57.74 & 57.57 & 59.95 & 60.31 & 60.13 & 63.23 & 63.61 & 63.42 & 60.29 & \\
\hline \multicolumn{2}{|c|}{ Mean $(T)$} & 60.11 & 60.47 & & & & & & & & & & & & \\
\hline \multicolumn{16}{|c|}{$\mathrm{CD}(p=0.05)$} \\
\hline \multicolumn{3}{|l|}{ Fruit (F) } & 0.035 & FxP & 0.061 & \multicolumn{2}{|c|}{ PxS } & 0.057 & \multicolumn{2}{|c|}{ FxTxS } & \multicolumn{3}{|l|}{0.103} & & \\
\hline \multicolumn{3}{|c|}{ Packing (P) } & 0.028 & FxT & 0.049 & & $x S$ & 0.045 & \multicolumn{2}{|c|}{ PxTxS } & 0.080 & & & & \\
\hline \multicolumn{3}{|c|}{ Temperature $(T)$} & 0.020 & FxS & 0.075 & & PxT & 0.089 & \multicolumn{2}{|c|}{ FXPXTXS } & 0.137 & & & & \\
\hline \multicolumn{3}{|c|}{ Storage (S) } & 0.029 & PxT & 0.038 & & PxS & 0.098 & & & & & & & \\
\hline
\end{tabular}

osmo-dried apple rings during storage has been reported by Sharma (1996).

Further, persimmon and pear fruit rolls were found rich in

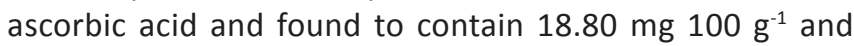

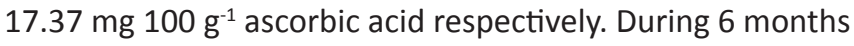
of storage studies the ascorbic acid decreased to $13.83 \mathrm{mg}$ $100 \mathrm{~g}^{-1}$ from $14.27 \mathrm{mg} 100 \mathrm{~g}^{-1}$, irrespective of fruit type and storage temperature (Table 3 ). Further, maximum decrease

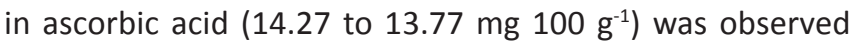
in fruit rolls packed in polyethylene pouches, whereas minimum decrease (14.27 to $13.88 \mathrm{mg} 100 \mathrm{~g} \mathrm{~g}^{-1}$ ) was observed in laminated pouches after 6 months of storage. Storage of rolls at refrigerated temperature also resulted in a minimum change in ascorbic acid after 6 months of storage (14.27 to $14.11 \%)$. The loss of ascorbic acid could be attributed to oxidation of ascorbic acid during the storage. Similarly, Mir (1990) also reported decrease in ascorbic acid in osmotically dehydrated apricot fruits.

A slight decrease in total phenolics content of fruit rolls was recorded in all the treatments during the storage (Table 4). Maximum phenol was recorded in persimmon (1509.14 mg $\left.100 \mathrm{~g}^{-1}\right)$ and minimum in pear $\left(272.36 \mathrm{mg} 100 \mathrm{~g}^{-1}\right)$, which after the six months of storage reduced to 1469.31 and $255.57 \mathrm{mg}$ $100 \mathrm{~g}^{-1}$, respectively. During 6 months of storage studies, the average initial value $\left(810.12 \mathrm{mg} 100 \mathrm{~g}^{-1}\right)$ of phenol decreased to

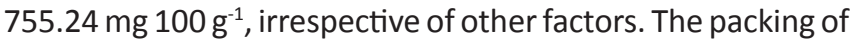
rolls in laminated pouches shows significant effect and found suitable for storage at low temperature. The minimal changes at refrigerated storage conditions might be due to the slower enzymatic reactions in dried products at lower temperature.

The data presented in Table 5 revealed that the peach and persimmon fruit roll contains carotenoid contents, which were found to decrease significantly during the storage (Table 5). 


\begin{tabular}{|c|c|c|c|c|c|c|c|c|c|c|c|c|c|c|c|}
\hline \multirow{3}{*}{$\begin{array}{l}\text { Packing } \\
\text { Material } \\
\text { (P) }\end{array}$} & \multirow[t]{3}{*}{$\mathrm{SP}$} & \multicolumn{12}{|c|}{ Fruit (F) } & \multirow{3}{*}{$\begin{array}{l}\text { Grand } \\
\text { mean }\end{array}$} & \multirow{3}{*}{$\begin{array}{c}\text { Mean } \\
(\mathrm{S})\end{array}$} \\
\hline & & \multicolumn{3}{|c|}{ Apple } & \multicolumn{3}{|c|}{ Peach } & \multicolumn{3}{|c|}{ Pear } & \multicolumn{3}{|c|}{ Persimmon } & & \\
\hline & & AT & RT & Mean & AT & RT & Mean & AT & RT & Mean & AT & RT & Mean & & \\
\hline \multicolumn{16}{|c|}{ Polyethylene } \\
\hline \multirow[t]{3}{*}{ Pouches } & 0 & 9.45 & 9.45 & 9.45 & 11.46 & 11.46 & 11.46 & 17.37 & 17.37 & 17.37 & 18.80 & 18.80 & 18.80 & 14.27 & 14.27 \\
\hline & 3 & 9.26 & 9.35 & 9.31 & 11.23 & 11.34 & 11.29 & 17.02 & 17.19 & 17.11 & 18.42 & 18.54 & 18.48 & 14.04 & 14.08 \\
\hline & 6 & 9.07 & 9.16 & 9.12 & 11.00 & 11.11 & 11.06 & 16.68 & 16.84 & 16.76 & 18.05 & 18.23 & 18.14 & 13.77 & 13.83 \\
\hline Mean & & 9.26 & 9.32 & 9.29 & 11.23 & 11.30 & 11.27 & 17.02 & 17.13 & 17.08 & 18.42 & 18.52 & 18.47 & 14.03 & \\
\hline \multirow[t]{3}{*}{ LP } & 0 & 9.45 & 9.45 & 9.45 & 11.46 & 11.46 & 11.46 & 17.37 & 17.37 & 17.37 & 18.80 & 18.80 & 18.80 & 14.27 & \\
\hline & 3 & 9.31 & 9.40 & 9.36 & 11.33 & 11.40 & 11.37 & 17.11 & 17.28 & 17.20 & 18.50 & 18.62 & 18.56 & 14.12 & \\
\hline & 6 & 9.12 & 9.21 & 9.17 & 11.12 & 11.32 & 11.22 & 16.76 & 16.93 & 16.85 & 18.12 & 18.48 & 18.30 & 13.88 & \\
\hline \multicolumn{2}{|l|}{ Mean } & 9.29 & 9.35 & 9.32 & 11.30 & 11.39 & 11.35 & 17.08 & 17.19 & 17.14 & 18.47 & 18.63 & 18.55 & 14.09 & \\
\hline \multicolumn{2}{|c|}{ Grand mean } & 9.28 & 9.34 & 9.31 & 11.27 & 11.35 & 11.31 & 17.05 & 17.16 & 17.11 & 18.45 & 18.58 & 18.51 & 14.06 & \\
\hline \multicolumn{2}{|l|}{ Mean $(T)$} & 14.01 & 14.11 & & & & & & & & & & & & \\
\hline \multicolumn{16}{|c|}{$\mathrm{CD}(p=0.05)$} \\
\hline \multicolumn{3}{|l|}{ Fruit (F) } & 0.089 & FxP & 0.13 & \multicolumn{2}{|c|}{ PxS } & 0.19 & \multicolumn{2}{|c|}{ FxTxS } & 0.32 & & & & \\
\hline \multicolumn{3}{|c|}{ Packing (P) } & 0.078 & FXT & 0.16 & & $x S$ & 0.15 & \multicolumn{2}{|c|}{ PxTxS } & NS & & & & \\
\hline \multicolumn{3}{|c|}{ Temperature $(T)$} & 0.064 & FxS & 0.24 & $\mathrm{Fx}$ & PxT & 0.28 & \multicolumn{2}{|c|}{ FxPxTxS } & NS & & & & \\
\hline \multicolumn{3}{|c|}{ Storage (S) } & 0.095 & PxT & NS & & PxS & NS & & & & & & & \\
\hline
\end{tabular}

Table 4: changes in total phenols $\left(\mathrm{mg} 100 \mathrm{~g}^{-1}\right)$ of single fruit rolls during storage

\begin{tabular}{|c|c|c|c|c|c|c|c|c|c|c|c|c|c|c|}
\hline \multirow[t]{3}{*}{ PM SP } & \multicolumn{12}{|c|}{ Fruit (F) } & \multirow{3}{*}{$\begin{array}{l}\text { Grand } \\
\text { mean }\end{array}$} & \multirow{3}{*}{$\begin{array}{c}\text { Mean } \\
\text { (S) }\end{array}$} \\
\hline & \multicolumn{3}{|c|}{ Apple } & \multicolumn{3}{|c|}{ Peach } & \multicolumn{3}{|c|}{ Pear } & \multicolumn{3}{|c|}{ Persimmon } & & \\
\hline & AT & RT & Mean & AT & RT & Mean & AT & RT & Mean & AT & RT & Mean & & \\
\hline
\end{tabular}

Polyethylene

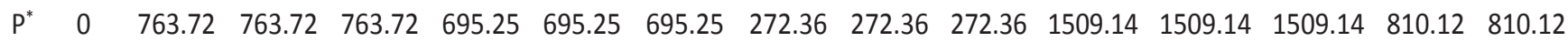

$\begin{array}{llllllllllllllll}3 & 730.36 & 746.54 & 738.45 & 665.40 & 674.56 & 669.98 & 251.69 & 266.49 & 259.09 & 1450.00 & 1471.46 & 1460.73 & 782.06 & 785.90\end{array}$

$\begin{array}{lllllllllllllll}6 & 696.44 & 717.69 & 707.07 & 624.16 & 644.96 & 634.56 & 226.74 & 239.46 & 233.10 & 1395.56 & 1440.16 & 1417.86 & 748.15 & 755.24\end{array}$

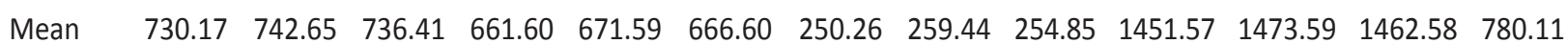

LP $\quad 0 \quad \begin{array}{llllllllllllll} & 763.72 & 763.72 & 763.72 & 695.25 & 695.25 & 695.25 & 272.36 & 272.36 & 272.36 & 1509.14 & 1509.14 & 1509.14 & 810.12\end{array}$

$\begin{array}{llllllllllllll}3 & 736.14 & 752.16 & 744.15 & 671.89 & 681.76 & 676.83 & 252.86 & 260.47 & 256.67 & 1475.16 & 1487.46 & 1481.31 & 789.74\end{array}$

$\begin{array}{llllllllllllll}6 & 711.68 & 731.19 & 721.44 & 640.21 & 660.51 & 650.36 & 234.49 & 245.14 & 239.82 & 1414.66 & 1460.72 & 1437.69 & 762.33\end{array}$

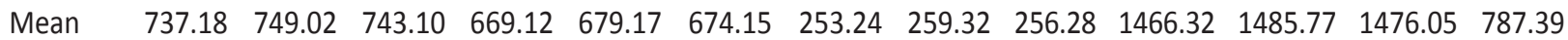

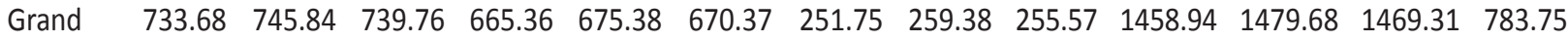

mean

Mean (T) $777.43 \quad 790.07$

$\mathrm{CD}(p=0.05)$

$\begin{array}{llllllll}\text { Fruit (F) } & 3.20 & \text { FxP } & 4.80 & \text { PxS } & 5.87 & \text { FxTxS } & 8.14\end{array}$

$\begin{array}{llllllll}\text { Packing (P) } & 4.45 & \text { FxT } & 6.30 & \text { TxS } & 6.32 & \text { PxTXS } & 7.23\end{array}$

$\begin{array}{llllllll}\text { Temperature (T) } & 3.57 & \text { FxS } & 4.65 & \text { FxPxT } & 7.11 & \text { FxPxTxS } & 3.45\end{array}$

$\begin{array}{llllll}\text { Storage (S) } & 5.85 & \text { PxT } & 7.64 & \text { FxPxS } & 7.93\end{array}$

PM: Packing Material (P); $P^{*}$ : Pouches 


\begin{tabular}{|c|c|c|c|c|c|c|c|c|c|}
\hline \multirow{3}{*}{$\begin{array}{l}\text { Packing } \\
\text { Material (P) }\end{array}$} & \multirow[t]{3}{*}{ SP } & \multicolumn{6}{|c|}{ Fruit (F) } & \multirow{3}{*}{$\begin{array}{l}\text { Grand } \\
\text { mean }\end{array}$} & \multirow{3}{*}{$\begin{array}{c}\text { Mean } \\
\text { (S) }\end{array}$} \\
\hline & & \multicolumn{3}{|c|}{ Peach } & \multicolumn{3}{|c|}{ Persimmon } & & \\
\hline & & AT & RT & Mean & AT & RT & Mean & & \\
\hline \multicolumn{10}{|l|}{ Polyethylene } \\
\hline \multirow[t]{3}{*}{ Pouches } & 0 & 1.86 & 1.86 & 1.86 & 9.60 & 9.60 & 9.60 & 5.73 & 9.60 \\
\hline & 3 & 1.67 & 1.71 & 1.69 & 8.64 & 8.81 & 8.73 & 5.21 & 8.90 \\
\hline & 6 & 1.49 & 1.52 & 1.51 & 7.68 & 7.83 & 7.76 & 4.63 & 7.93 \\
\hline Mean & & 1.67 & 1.70 & 1.69 & 8.64 & 8.75 & 8.69 & 5.19 & \\
\hline \multirow[t]{3}{*}{ LP } & 0 & 1.86 & 1.86 & 1.86 & 9.60 & 9.60 & 9.60 & 5.73 & \\
\hline & 3 & 1.73 & 1.78 & 1.76 & 8.93 & 9.20 & 9.07 & 5.41 & \\
\hline & 6 & 1.54 & 1.59 & 1.57 & 7.97 & 8.21 & 8.09 & 4.83 & \\
\hline Mean & & 1.71 & 1.74 & 1.73 & 8.83 & 9.00 & 8.92 & 5.32 & \\
\hline Grand mean & & 1.69 & 1.72 & 1.71 & 8.74 & 8.88 & 8.80 & 5.26 & \\
\hline Mean $(\mathrm{T})$ & & 5.27 & 5.37 & & & & & & \\
\hline \multicolumn{10}{|l|}{$\mathrm{CD}(p=0.05)$} \\
\hline Fruit (F) & & & 0.23 & FxP & 0.37 & PxS & 0.39 & FxTxS & 0.62 \\
\hline Packing (P) & & & 0.19 & FxT & 0.31 & TxS & 0.29 & PxTxS & 0.52 \\
\hline Temperature $(\mathrm{T})$ & & & 0.17 & FxS & 0.45 & FxPxT & 0.51 & FxPxTxS & 0.98 \\
\hline Storage (S) & & & 0.20 & PxT & 0.25 & FxPxS & 0.81 & & \\
\hline
\end{tabular}

Maximum carotenoids were recorded in persimmon $(9.60$ $\mathrm{mg} 100 \mathrm{~g} \mathrm{~g}^{-1}$ ) and minimum in peach (1.86 mg $\left.100 \mathrm{~g}^{-1}\right)$, which

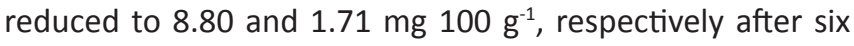
months of storage. During 6 months storage, the average initial value $\left(9.60 \mathrm{mg} 100 \mathrm{~g}^{-1}\right)$ of total carotenoids decreased

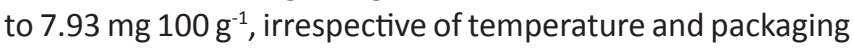
material. Further, products packed in laminated pouches retained more carotenoids than those in polyethylene pouches. Low storage temperature also shows significant effect as rolls showed a less change in total carotenoids (9.60

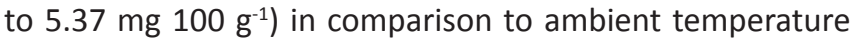
( 9.60 to $5.27 \mathrm{mg} 100 \mathrm{~g}^{-1}$ ). This might be due to impervious nature of laminates to air and water which might be helpful in prevention of oxidation of carotenoids. Gahilad et al. (1982) also reported a reduction in carotenoids content in mango leather during 70 days storage in polyethylene pouches.

The free radical scavenging activity (Table 6) were in the range of 60.61 to $88.52 \%$ in different fruit rolls with highest activity in persimmon (88.52\%) and lowest in pear (60.61 per cent). During 6 months of storage studies, the average initial free radical scavenging activity $(73.10 \%)$ decreased to $67.18 \%$, irrespective of other factors. Further, maximum decrease in free radical scavenging activity (73.10 to $66.45 \%$ ) observed in fruit rolls packed in polyethylene pouches with minimum decrease (73.10 to $67.92 \%$ ) in laminated pouches after 6 months of storage. Low temperature stored fruit rolls showed a less change in free radical scavenging activity. According to Goyal and Khetarpaul (1994); Negi et al. (2001), Sato et al.
(2006); Turkmen et al. (2006), the foods undergo numerous processing changes before consumption and may alter their nutritional profile as well as antioxidants contents.

\subsection{Equilibrium relative humidity $(E R H)$ of single fruit rolls}

Data presented in Figure 1 on moisture sorption behaviour revealed that the moisture absorbed by single fruit rolls held at different relative humidity (0 to $100 \%$ ) caused the deterioration of single fruit rolls. The single fruit rolls with initially attractive colour and good texture turned dark soft textured, hard textured and/or mouldy with increase in relative humidity. The critical points during storage of single fruit rolls were found to be at $60 \%$ with the equilibrium moisture content of $15.21 \%, 15.19 \%, 9.98 \%$ and $12.48 \%$ in apple, peach, pear and persimmon fruit rolls, respectively.

\subsection{Sensory evaluation}

The fruit rolls were evaluated on the basis of sensory and the overall acceptability score in single fruit rolls have been presented in the Table 7. Overall acceptability was in the range of 7.10 to 7.60 in different fruit rolls. Maximum overall acceptability was recorded in peach (7.60) and minimum in persimmon rolls (7.10), which after the six months of storage reduced to 6.98 and 6.51 , respectively. During 6 months of storage, the average initial score value (7.30) of overall acceptability decreased to 6.04 , irrespective of other factors. Among the packaging, maximum decrease in overall acceptability (7.30 to 5.88) was observed in polyethylene pouches, whereas minimum (7.30 to 6.21) was observed in 


\begin{tabular}{|c|c|c|c|c|c|c|c|c|c|c|c|c|c|c|c|}
\hline \multirow{3}{*}{$\begin{array}{l}\text { Packing } \\
\text { Material } \\
\text { (P) }\end{array}$} & \multirow[t]{3}{*}{ SP } & \multicolumn{12}{|c|}{ Fruit (F) } & \multirow{3}{*}{$\begin{array}{l}\text { Grand } \\
\text { mean }\end{array}$} & \multirow{3}{*}{$\begin{array}{c}\text { Mean } \\
\text { (S) }\end{array}$} \\
\hline & & \multicolumn{3}{|c|}{ Apple } & \multicolumn{3}{|c|}{ Peach } & \multicolumn{3}{|c|}{ Pear } & \multicolumn{3}{|c|}{ Persimmon } & & \\
\hline & & AT & RT & Mean & AT & RT & Mean & AT & RT & Mean & AT & RT & Mean & & \\
\hline \multicolumn{16}{|c|}{ Polyethylene } \\
\hline \multirow[t]{3}{*}{ Pouches } & 0 & 78.00 & 78.00 & 78.00 & 65.26 & 65.26 & 65.26 & 60.61 & 60.61 & 60.61 & 88.52 & 88.52 & 88.52 & 73.10 & 73.10 \\
\hline & 3 & 74.10 & 75.58 & 74.84 & 62.00 & 63.24 & 62.62 & 57.58 & 58.73 & 58.16 & 84.09 & 85.78 & 84.94 & 70.14 & 70.68 \\
\hline & 6 & 70.20 & 71.60 & 70.90 & 58.73 & 59.91 & 59.32 & 54.55 & 55.64 & 55.10 & 79.67 & 81.26 & 80.47 & 66.45 & 67.18 \\
\hline \multicolumn{2}{|l|}{ Mean } & 74.10 & 75.06 & 74.58 & 62.00 & 62.80 & 62.40 & 57.58 & 58.33 & 57.95 & 84.09 & 85.19 & 84.64 & 69.89 & \\
\hline \multirow[t]{3}{*}{ LP } & 0 & 78.00 & 78.00 & 78.00 & 65.26 & 65.26 & 65.26 & 60.61 & 60.61 & 60.61 & 88.52 & 88.52 & 88.52 & 73.10 & \\
\hline & 3 & 74.88 & 77.13 & 76.01 & 62.65 & 64.53 & 63.59 & 58.19 & 59.93 & 59.06 & 84.98 & 87.53 & 86.26 & 71.23 & \\
\hline & 6 & 71.76 & 73.20 & 72.48 & 60.04 & 61.24 & 60.64 & 55.76 & 56.88 & 56.32 & 81.44 & 83.07 & 82.26 & 67.92 & \\
\hline \multicolumn{2}{|l|}{ Mean } & 74.88 & 76.11 & 75.50 & 62.65 & 63.68 & 63.16 & 58.19 & 59.14 & 58.66 & 84.98 & 86.37 & 85.68 & 70.75 & \\
\hline \multicolumn{2}{|c|}{ Grand mean } & 74.49 & 75.59 & 75.04 & 62.32 & 63.24 & 62.78 & 57.88 & 58.73 & 58.31 & 84.54 & 85.78 & 85.16 & 70.32 & \\
\hline \multicolumn{2}{|c|}{ Mean $(T)$} & 69.81 & 70.83 & & & & & & & & & & & & \\
\hline \multicolumn{16}{|c|}{$\mathrm{CD}(p=0.05)$} \\
\hline \multicolumn{3}{|l|}{ Fruit (F) } & 0.22 & FxP & 0.36 & \multicolumn{2}{|c|}{ PxS } & 0.55 & \multicolumn{2}{|c|}{ FxTxS } & \multicolumn{3}{|l|}{0.68} & & \\
\hline \multicolumn{3}{|c|}{ Packing (P) } & 0.40 & FxT & 0.54 & T) & xS & 0.41 & PxT & TXS & 0.60 & & & & \\
\hline Tempera & ture ( & & 0.29 & FxS & 0.41 & Fx & PXT & 0.51 & FxPx & XTXS & 0.31 & & & & \\
\hline Storage & & & 0.51 & PxT & 0.64 & $\mathrm{Fx}$ & xS & 0.60 & & & & & & & \\
\hline Table 7: & Effect & of stor & ge on $c$ & verall ac & cceptabi & ility* o & sle tr & it rolls & & & & & & & \\
\hline Packing & $\mathrm{SP}$ & & & & & & Frui & it (F) & & & & & & Grand & Mean \\
\hline Material & & & Apple & & & Peach & & & Pear & & & ersimm & & mean & \\
\hline & & AT & RT & Mean & AT & RT & Mean & AT & RT & Mean & AT & RT & Mean & & \\
\hline Polyethy & lene & & & & & & & & & & & & & & \\
\hline Pouches & 0 & 7.30 & 7.30 & 7.30 & 7.60 & 7.60 & 7.60 & 7.20 & 7.20 & 7.20 & 7.10 & 7.10 & 7.10 & 7.30 & 7.30 \\
\hline & 3 & 6.50 & 6.70 & 6.60 & 6.80 & 7.00 & 6.90 & 6.40 & 6.60 & 6.50 & 6.30 & 6.50 & 6.40 & 6.60 & 6.78 \\
\hline & 6 & 5.80 & 6.00 & 5.90 & 6.00 & 6.20 & 6.10 & 5.70 & 5.90 & 5.80 & 5.60 & 5.80 & 5.70 & 5.88 & 6.04 \\
\hline Mean & & 6.53 & 6.67 & 6.60 & 6.80 & 6.93 & 6.87 & 6.43 & 6.57 & 6.50 & 6.33 & 6.47 & 6.40 & 6.59 & \\
\hline LP & 0 & 7.30 & 7.30 & 7.30 & 7.60 & 7.60 & 7.60 & 7.20 & 7.20 & 7.20 & 7.10 & 7.10 & 7.10 & 7.30 & \\
\hline & 3 & 6.90 & 7.00 & 6.95 & 7.20 & 7.30 & 7.25 & 6.80 & 6.90 & 6.85 & 6.70 & 6.80 & 6.75 & 6.95 & \\
\hline & 6 & 6.20 & 6.30 & 6.25 & 6.40 & 6.50 & 6.45 & 6.10 & 6.20 & 6.15 & 6.00 & 6.00 & 6.00 & 6.21 & \\
\hline Mean & & 6.80 & 6.87 & 6.83 & 7.07 & 7.13 & 7.10 & 6.70 & 6.77 & 6.73 & 6.60 & 6.63 & 6.62 & 6.82 & \\
\hline Grand $m$ & ean & 6.67 & 6.77 & 6.72 & 6.93 & 7.03 & 6.98 & 6.57 & 6.67 & 6.62 & 6.47 & 6.55 & 6.51 & 6.71 & \\
\hline Mean ( $T$ & & 6.66 & 6.75 & & & & & & & & & & & & \\
\hline $\mathrm{CD}(p=0$. & & & & & & & & & & & & & & & \\
\hline Fruit (F) & & & 0.025 & FxP & 0.037 & $P$ & $\mathrm{XS}$ & 0.040 & FxT & TXS & 0.059 & & & & \\
\hline Packing & & & 0.016 & FXT & 0.030 & T) & $\mathrm{KS}$ & 0.030 & PXT & TXS & 0.050 & & & & \\
\hline Tempera & ture ( & & 0.011 & FxS & 0.039 & $\mathrm{Fx}$ & PXT & 0.049 & FxPx & $x T x S$ & 0.117 & & & & \\
\hline Storage & & & 0.022 & PxT & 0.021 & $\mathrm{Fx}$ & PXS & 0.060 & & & & & & & \\
\hline
\end{tabular}


Apple fruit rolls $-\square$ - Peach fruit rolls $-\triangle$ Pear fruit rolls $X$ Persimmon fruit rolls

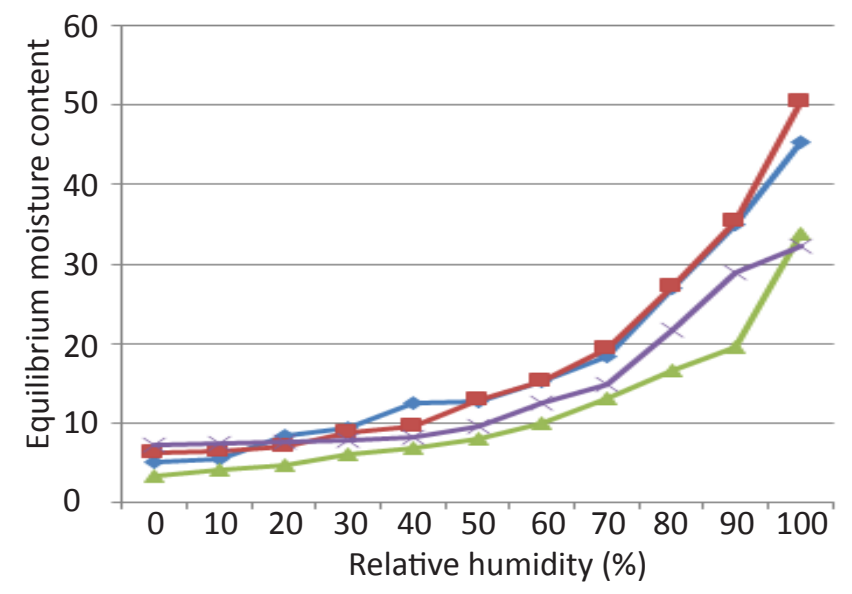

Figure 1: Curve for equilibrium relative humidity of apple, peach, pear and persimmon fruit rolls

laminated pouches packed samples after 6 months of storage. Samples packed in polyethylene pouches and stored at ambient temperature showed a maximum decrease in overall acceptability by interaction of factors, while the samples packed in laminated pouches and stored at refrigerated temperature resulted in a minimum change in overall acceptability after 6 months of storage. Low temperature stored fruit samples showed a slight change in overall acceptability. The decrease in score for colour, taste, texture and overall acceptability were less in products packed in laminated pouches as compared to polyethylene pouches. This trend might be due to impervious nature of the laminated pouches which provide a barrier to light and air. The deteriorative changes in flavour, texture and colour initiates during drying and continues in the storage (Sagar et al., 1999).

\section{Conclusion}

On the basis of chemical characteristics, nutritional value and minimal changes in quality parameters during storage, the packing of fruit rolls in laminated pouches followed by storage under refrigerated conditions $\left(4-5^{\circ} \mathrm{C}\right)$ has been optimized for better storage of fruit rolls. The aluminium laminated pouches acts as a moisture barrier and thus helps in retaining the texture and overall acceptability of rolls during storage. Further, the low temperature also found suitable for retention of quality characteristics of fruit rolls. Thus, the packing of fruit rolls in laminated pouches and storage under refrigerated is optimized for storage of fruit rolls.

\section{References}

Ambrose, D.C.P., Sreenarayana, V.V., 1998. Studies on dehydration of garlic. Journal of Food Science and Technology 35(3), 242-244.

Amerine, M.A., Pangborn, R.M., Rossler, E.B., 1965. Principles of sensory evaluation of food. Academic Press, New
York, 254.

AOAC, 1995. Official methods of analysis of association of official analytical chemists, 16th edition. Vol. I and II. Association of Official Analytical Chemists, Arlington, Virginia, USA.

Bhardwaj, J.C., Lal, B.B., 1990. Study on drying behaviour of rings from different apple cultivars of Himachal Pradesh. J. Federation Sci. Technol., 27(3), 144-149.

Brand Williams W, Cuvelier M E and Berset C. 1995. Use of a Free Radical Method to evaluate antioxidant activity. Lebensm-Wiss u-Technology 28, 25-30.

Bray, H.G., Thorpe, W.V., 1954. Standard methods of biochemical analysis. Thimmaiah, S.K. (Ed.). Kalyani Publishers, New Delhi, 287-288.

Chan, H.T., Caveletto, C.G., 1987. Dehydration and storage study of papaya leather. Journal of Food Science and Technology 30(6), 457-458.

Che, M.Y.B., Taufik, Y.C.M., 1995. Development and stability of jack fruit leather. Tropical Science 35, 245-250.

Chinnici, F., Bendini, A., Gaiani, A., Riponi, C., 2004. Radical scavenging activities of peels and pulps from cv. Golden Delicious apples as related to their phenolic composition. Journal of Agricultural and Food Chemistry 52(15), 4684-4689.

Gahilad, A.T., Pawar, V.N., Khedhar, D.M., Jawhar, S.J., 1982. Suitability of mango varieties for processing mango leather. Abstracts of technical paper. Proceedings International Food Conference, 82.

Goyal, R., Khetarpaul, N., 1994. Preparation and fermentation of rice-defatted soya flour blends: effect on protein, fat and ash content. Plant Food and Human Nutrition 45, 81-89.

Irwandi, J., Che, M.Y.B., Yusof, S., Jinap, S., Sugisawa, H., 1998. Effects of type of packaging materials on physicochemical, microbiological and sensory characteristics of durian fruit leather during storage. Journal of the Science of Food and Agriculture 76, 427-434.

Krishnaveni, A., Manimegalai, G., Vennila, P., Sarvanakumar, R., 1999. Storage stability of jackfruit bar in different packaging materials. Indian Food Packer 53(6), 67-71.

Kumar, R., 1999. Dehydration in apricot (Prunus armeniaca L.) grown under mid-hill conditions of Himachal Pradesh. M. Sc. Thesis, Dr. Y.S. Parmar University of Horticulture and forestry, Solan, Himachal Pradesh, India.

Lane, J.H., Eynon, L., 1923. Determination of reducing sugars by Fehling's solution with methylene blue as an indicator. Journal of the Society of Chemical Industry 42, 32.

Mir, M.A., 1990. Development and evaluation of fortified mango bars. Ph.D. Thesis, G.B. Pant University of Agriculture and Technology, Pantnagar, India.

Mishra, H.N., Jacob, J.K., Srinivasan, N., 2002. Preparation of apple powder and evaluation of its shelf-life. Bev. Food World 29(1), 49-52.

Negi, A., Boora, P., Khetarpaul, N., 2001. Starch and protein 
digestibility of newly released moth beans cultivars: effect of soaking, dehulling, germination and pressure cooking. Nahrung 45, 251-254.

Panse, V.P., Sukhatme, P.V., 1967. Statistical methods for agricultural workers, Indian Council of Agricultural Research, New-Delhi, 70-72.

Ranganna, S., 1997. Handbook of analysis and quality control for fruit and vegetable products. ( $2^{\text {nd }} \mathrm{Edn}$.). Tata McGraw Hill Publishing Company Ltd., New Delhi, India, 1109.

Sagar, V.R., Khurdiya, D.S., 1999. Studies on dehydration of Dashehri mango slices. Indian Food Packer 53(1), 5-9.

Sagar, V.R., Khurdiya, D.S., Balakrishnan, M., 1999. Quality of dehydrated ripe mango slices as affected by packaging material and mode of packaging. Journal of Food Science and Technology 36(1), 67-70.

Sato, E., Kohno, M., Hamano, H., Niwano, Y., 2006. Increased antioxidative potency of garlic by spontaneous short term fermentation. Plant Food and Human Nutrition 61, 157-160.

Sharma, K.D., Sethi, V., Maini, S.B., 2002. Effect of pertreatment and package on chemical and sensory characteristics of dried apples. Indian Food Packer. 54(1), 52-59.
Sharma, K.D., 1996. Osmo-processing studies in apple grown under different agro-climatic conditions of Himachal Pradesh. Ph.D. Thesis, Indian agricultural Research Institute, New Delhi, India.

Sharma, K.D., Chandel, V., Verma, A.K., Gupta, A., 2014. Development and evaluation of functionally enriched fruit rolls. Indian Food Packer 68(4), 30-40.

Thakur, N., 1997. Development of apricot soya products and their quality evaluation. M.Sc. Thesis, Dr. Y. S. Parmar University of Horticulture and Forestry, Nauni, Solan, India.

Turkmen, N., Poyrazoglu, E.S., Sari, F., Velioglu, Y.S., 2006. Effect of cooking methods on chlorophylls, pheophytins and colour of green leafy vegetables. International Journal of Food Science \& Technology 41, 281-288.

Vijayanand, P., Yadav, A.R., Balasubramanyam, N., Narasimham P., 2000. Storage stability of guava fruit bar prepared using a new process. LWT-Food Science and Technology 33, 132-137.

Wink, W.A., 1946. Wink's weight equilibrium method for $\mathrm{ERH}, 480$. 